Stewart, T. R., \& Mumpower, J. L. (2004). Detection and Selection Decisions in the Practice of Screening Mammography. Journal of Policy Analysis and Management, 23(4), 908-920.

\title{
DETECTION AND SELECTION DECISIONS IN THE PRACTICE OF SCREENING MAMMOGRAPHY
}

Thomas R. Stewart and Jeryl L. Mumpower

A large class of problems in society requires detection or selection decisions. Examples include: Who should receive extra scrutiny in airport screening? What personal characteristics, if any, should patrolling police attend to? What blood alcohol levels constitute driving while intoxicated? What thresholds should be used for issuing severe weather warnings or terrorist-related security alerts? At what age, if any, should men routinely receive PSA testing for prostate cancer, and what thresholds should be established for treatment?

In this paper we focus on an important member of this class-the practice of screening mammography. Substantial uncertainty and disagreement persist concerning the value of regular mammogram screenings for women, particularly those between the ages of 40 and 49. For example, the National Breast Cancer Coalition (2003) has concluded that there is insufficient evidence to recommend for or against screening mammography for any age group of women. Conversely, the U.S. Preventive Services Task Force (2002) has recommended screening mammography, with or without clinical breast examination, every 1 to 2 years for women aged 40 and older. ${ }^{1}$

The results of seven controlled clinical trials on the effectiveness of screening mammography remain controversial (e.g., de Koning, 2003; Olsen and Gotzsche, 2001). Critics argue that the studies are flawed and inconclusive. Taken as a whole, data from these trials indicate a 24 percent decline in breast cancer mortality associated with

\footnotetext{
${ }^{1}$ As an anonymous reviewer noted, the Task Force was rather tepid in its endorsement. Their summary of recommendations concluded: "For women age 40-49, the evidence that screening mammography reduced mortality from breast cancer is weaker, and the absolute benefit of mammography is smaller than it is for older women."
} 
mammography. Despite the large number of women enrolled in clinical studies of mammography effectiveness, meta-analysis indicates that the estimated protective effects of mammography depend strongly upon the inclusion or exclusion of specific contested studies. Olsen and Gotszche (2001) argue that the principal studies most favorable to mammography display systematic prior differences between the mammography and non-mammography comparison group that undermine study validity. The exclusion of such studies leads to no statistically significant benefit associated with the intervention. Most discouraging, large increases in the proportion of women receiving mammography have not resulted in large declines in breast cancer mortality.

\section{OVERVIEW OF MAMMOGRAPHY SCREENING AND PRACTICE}

Figure 1 provides a framework for describing the relations among three domains of decision making about mammography screening: decisions by women and their doctors to obtain screening, decisions by radiologists to recommend biopsy, and decisions by policymaking bodies to recommend and support screening for certain sub-groups of women.

Beginning at the left, some fraction of the eligible women voluntarily decides to be screened. ${ }^{2}$ Their decision may be influenced by the recommendations of influential organizations, by their doctor, by their families and friends, and by information they receive about mammography. Recently there has been substantial effort to increase the proportion of women who decide to be screened. Some studies suggest that many women are confused and are not well-informed decisionmakers (Rimer et al., 2002; Schwartz et al., 1997).

Next, screening takes place, and the radiologist's decisions are either positive (biopsy recommended) or negative (normal follow-up). ${ }^{3}$ At this stage, the base rate of women with malignancies, the accuracy of interpretation of mammograms, and the thresholds for choosing between a positive or negative finding are important considerations.

There are four possible outcomes of the radiologist's decision. If the preliminary diagnosis is positive, a biopsy is obtained; the biopsy result may then indicate a malignant (true positive) or benign (false positive) condition. Data show that there are about three false positives for every true positive. If the finding is negative, there is no biopsy. While most of the women who are not recommended for biopsy do not have a malignant mass (true negative), some do (false negative).

Each of the outcomes has associated costs and benefits. A true positive may mean earlier identification and treatment of cancer, possibly resulting in a better outcome. Mammographers want to avoid false negatives because undetected and untreated cancer is the worst possible outcome. Because of the high uncertainty, avoiding false negatives results in many false positives (biopsies that turn out to be negative). The appropriate tradeoff between false positives and false negatives is an important factor in the mammography debate.

Cost-effectiveness analyses (e.g., Salzmann, Kerlikowske, and Phillips, 1997) use information about uncertainty, costs, and effectiveness of various outcomes to evaluate alternative options, and these analyses influence recommendations made by organizations such as the National Cancer Society and the National Breast Cancer

\footnotetext{
${ }^{2}$ This is simplified, of course; women actually have a wider range of options for detecting breast cancer. ${ }^{3}$ Again, this is a simplification because radiologists have other options as well. We use the term "radiologist" rather than "mammographer" because not all radiologists who read mammograms are board certified mammographers.
} 


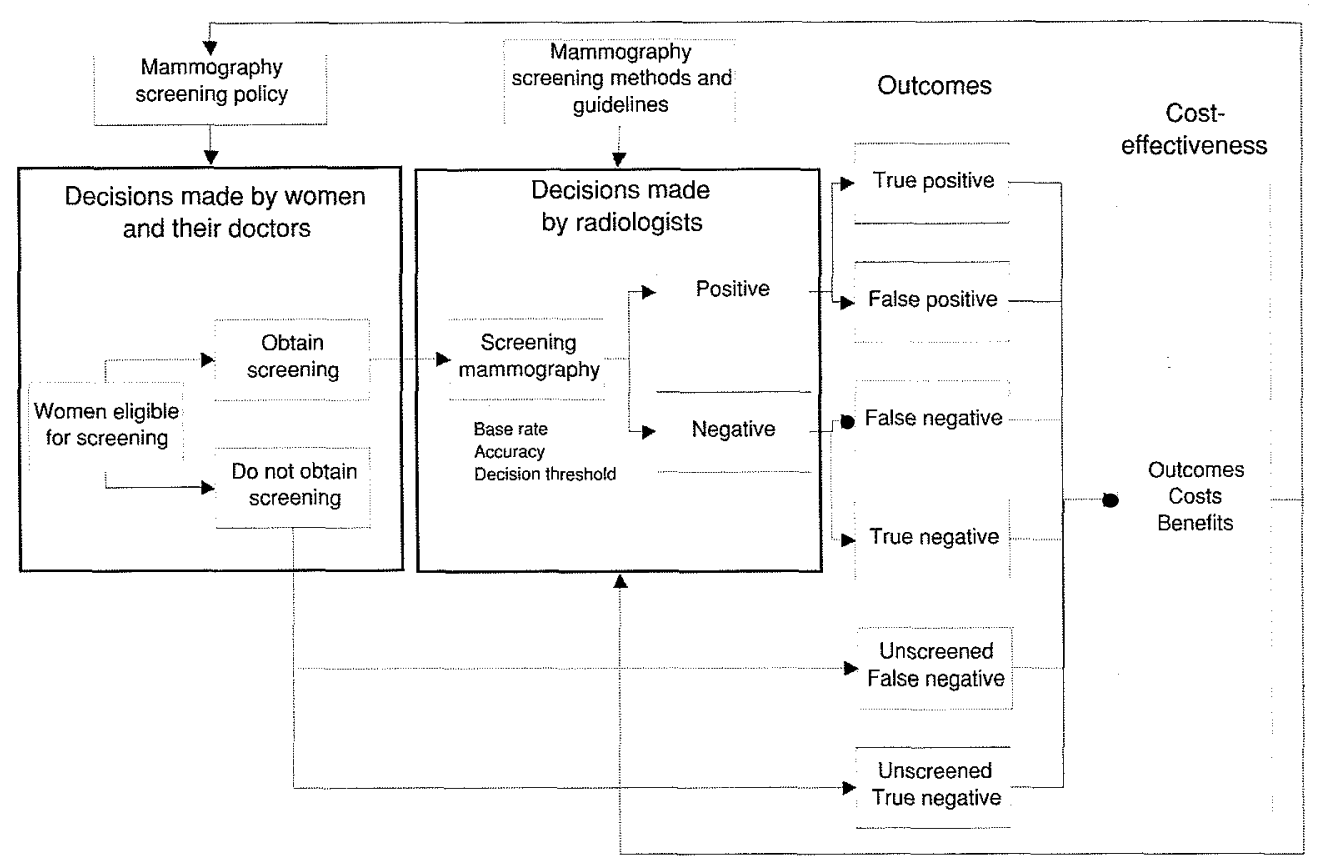

Figure 1. Schematic view of mammography screening.

Coalition and advisory bodies such as the U.S. Preventive Services Task Force. Cost effectiveness analyses, along with other information, influence the doctor's recommendations regarding mammography for their patients, as well as the decisions that radiologists make.

The focus of this paper is the decisions made by radiologists in their practice. These decisions not only occupy a central location in Figure 1, they are central to all issues surrounding screening mammography. While cost-effectiveness analyses and policy debates often assume that the accuracy of radiologists' judgments is fixed, research results strongly suggest that the interpretation of mammograms is less accurate than it could be and that there is wide variation among radiologists regarding the appropriate tradeoff between false positive and false negatives.

Addressing two critical "what if" questions can help inform the crucial decision makers: women, their doctors, and radiologists:

1. What if radiologists selected more or fewer women for biopsy?

2. What if interpretation of mammographic images was more accurate?

\section{A MODEL FOR ADDRESSING DETECTION AND SELECTION PROBLEMS}

Selection and detection problems often involve high-consequence decisions under substantial uncertainty. Decisionmakers face two kinds of errors, each with potentially serious consequences: false positives (falsely selecting a case) and false negatives (missing a correct selection). Since uncertainty creates an inevitable tradeoff between these two errors, the decision strategy must be based on values. 
Analysis alone cannot solve the problem, but it exposes the nature of the tradeoffs involved and can assure that decisionmakers consider all the possible outcomes. For related discussions see, for example, Hammond, Harvey, and Hastie (1992); Mumpower, Nath, and Stewart (2002); and Swets, Dawes, and Monahan (2000). The critical elements of these problems are base rate, uncertainty, decision threshold, and outcomes.

\section{Base Rate}

Among a group of women who have screening mammography, what proportion has malignancies? The base rate depends on age and on the time period considered. The relevant base rate for screening decisions is the probability of a malignancy among undiagnosed women at the time of screening (rather than the lifetime probability of breast cancer). The relevant base rate is for a period of 1 to about 5 years, depending on the screening interval. For any given screening technology, the base rate is critical in shaping the positive predictive value (proportion of true positives to all positives) and the negative predictive value (proportion of true negatives to false negatives) of the screening. All else being equal, low base rates imply low positive predictive values. Even very specific tests yield a large number of false negatives when compared with the small number of true detected cases of breast cancer within the population.

It is difficult to obtain an accurate base rate for a specific population, but we know it is low. Annual incidence of breast cancer is 0.001 to 0.006 , depending on age and other factors (Ries et al., 2003).

\section{Uncertainty}

Uncertainty is introduced by the inaccuracy of screening mammography. Images vary in quality depending on breast density and other factors, and features that can suggest cancer are often small or difficult to distinguish from normal tissue. Radiologists must therefore make judgments when they evaluate mammograms. Mushlin, Kouides, and Shapiro (1998) conducted a meta-analysis of published studies on the accuracy of screening mammography. They reported true positive rates (correct positive results/total number of patients with cancer), ranging from 0.83 to 0.95 ; the medical decision-making literature typically refers to the true positive rate as the sensitivity of a test. False positive rates (incorrect positive results/total number of patients without cancer) were reported from 0.009 to 0.065 ; the medical decision-making literature typically refers to one minus the false positive rate as the specificity of a test. A review by Woolf (2001) found that mammograms miss 0.12 to 0.37 of cancers, depending on the type and stage of cancer, the density of breast tissue, and other factors.

A study by Beam, Layde, and Sullivan (1996) of 108 radiologists from 50 centers found a range of at least 0.53 ( 0.47 to 1.00$)$ among radiologists in screening sensitivity (the ability to detect cancer when it is in fact present). ROC curve areas $\left(\mathrm{A}_{z}-\right.$ a measure of accuracy for which a value of 1 indicates perfect accuracy and 0.5 indicates accuracy no better than chance) ranged from 0.74 to 0.95 .

\section{Decision Threshold}

After examining mammographic images, the radiologist forms an opinion of "suspicion" or "likelihood" of malignancy on some continuum. In other words, the radiologist makes a continuous judgment, but the decision options are discrete (e.g., normal follow-up, return for screening in three months, obtain biopsy). Some way of converting continuous judgments to discrete actions is needed. Generally; it is 
assumed that this conversion involves one or more thresholds. If the judgment is higher than some threshold, then biopsy is recommended. Otherwise, no biopsy is recommended. This model is described in detail by Pauker and Kassirer (1980). The threshold may not be determined or even stated explicitly, but it is implicit in the radiologist's decisions.

In effect, the radiologist has to decide what level of suspicion warrants action. The threshold determines the selection rate, that is, the number of mammograms that are selected for biopsy or more frequent screening. A lower threshold means that more mammograms are selected. Furthermore, as is demonstrated below, the threshold influences the relative numbers of false positives and false negatives. Hence, thresholds should reflect the relative costs of these errors.

Different mammographers have different thresholds. Beam, Layde, and Sullivan (1996) found a range of at least 0.45 among mammographers in the proportion of women without breast cancer who were recommended for biopsy. Berg et al. (2000, 2002) found substantial disagreement both about features and about management decisions, and Elmore et al. (2002) found a wide range of false positive rates among mammographers. It is unclear whether this variation indicates differences in values, differences in base rates, differences in accuracy, use of inappropriate thresholds, or a combination of these factors.

\section{Outcomes}

Screening mammography is a detection problem with a low base rate, high uncertainty, and varying decision thresholds determined by physicians. These interact to determine the outcomes. The four possible outcomes of screening mammography were shown in Figure 1.

False positives-defined as women who are referred for biopsies or other additional diagnostic treatments but who are later found not to have tumors-are clearly the most frequent error. After 10 years, 50 percent of women who have annual screening could have a false positive result (Elmore et al., 1998). More than 80 percent of women who have suspicious mammograms have no cancer (National Breast Cancer Coalition, 2003). This leads to many negative biopsies and possibly to over-treatment, because, for example, a positive biopsy indicates the presence of cancer, but does not mean it will spread. False negatives are much less frequent, but potentially more serious, because they may result in an aggressive malignancy going untreated. Both false positives and false negatives depend on the selection rate, but they change in different directions. Figure 2 illustrates the tradeoff between false positives and false negatives.

This is an example based on the data presented in Table 1. Different base rates and different levels of uncertainty will result in different graphs, but the basic relations remain the same.

As the selection rate (the percentage of screened women who are diagnosed as positive) increases, the false negative proportion drops, but the false positive proportion increases. By increasing the selection rate, a certain number of false negatives are eliminated, at a cost of creating a number of false positives. ${ }^{4}$

\footnotetext{
${ }^{4}$ The present analysis relies on a key assumption that mammograms can be ranked by mammographers in terms of their suspiciousness, so that increasing the selection rate implies that the mammographer will refer an increasing proportion of the "next-most suspicious" mammograms for biopsy or other diagnostic treatment. Decisions about whether, for whom, and how often mammography should be deployed will result in changes in the frequency and relative proportions of the four possible outcome cells.
} 


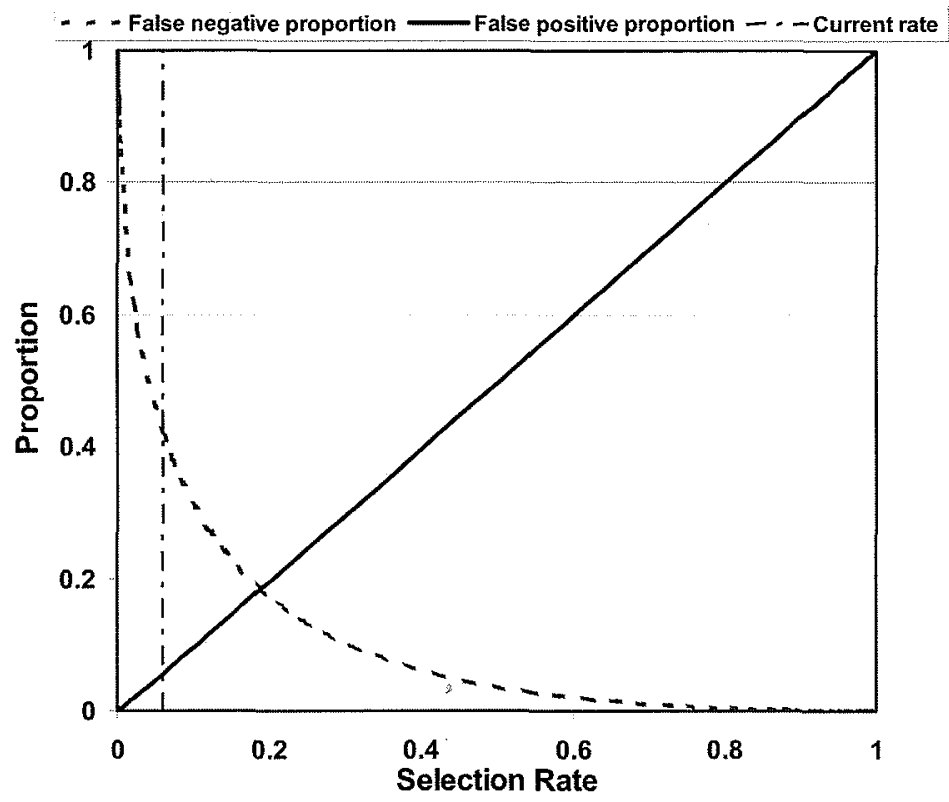

This is an example based on the data presented in Table 1. Different base rates and different levels of uncertainty will result in different graphs, but the basic relations remain the same.

Figure 2. Proportion of error as a function of selection rate.

Table 1. Results for women under 50 .

\begin{tabular}{llrrr}
\hline & & \multicolumn{2}{c}{ Decision } & \\
& & Negative & Positive & Total \\
\hline Biopsy & Malignant & 36 & 50 & 86 \\
result & Non-malignant & 9,360 & 554 & 9,914 \\
& Total & 9,396 & 604 & 10,000 \\
\hline
\end{tabular}

Based on Kolb, Lichy, and Newhouse (2002). Sample size adjusted to 10,000 for ease of interpretation.

The rate at which false positives are substituted for true positives at a given selection rate is called the marginal substitution rate. The optimal selection rate is found when the marginal substitution rate equals the ratio of the benefit of a true positive to the cost of a false positive. The optimal selection rate depends on the benefits of true positives (e.g., the health benefits of early detection and treatment) and true negatives (e.g., the psychological benefits of correct disease-free diagnoses) and the costs of false positives (e.g., costly and painful biopsies and other diagnostic treatments of healthy women) and false negatives (e.g., all the costs associated with failure to treat non-detected tumors). 


\section{THE PRACTICE OF MAMMOGRAPHY: DATA FROM ONE MAMMOGRAPHER}

During a study that lasted almost 6 years (January 15, 1995 to September 30, 2000), Kolb, Lichy, and Newhouse (2002) recorded data on 27,825 screenings of 11,130 asymptomatic women (women were screened more than once) at New York's Columbia-Presbyterian Medical Center. All were patients of one doctor (Kolb). The patients underwent mammography, physical exam, and ultrasound. Presence of breast cancer was determined by biopsy. Absence was determined by negative results for all screenings. Table 1 is based on 5826 screenings of women under 50 .

From Table 1, we can calculate a base rate of 0.0086 and a selection rate of 0.0604 . The false positive proportion is $554 / 9914=0.056$ and the false negative proportion is $36 / 86=0.419$. The vertical line in Figure 2 roughly represents these data. Note that although the false negative proportion is higher than for false positives, there are many more negatives than positives (due to the low base rate), resulting in over 15 false positives for every false negative.

The imputed marginal rate of substitution for this case is $33.2 .{ }^{5} \mathrm{~A}$ small increase in the selection rate would result in about 33 additional false positives for every true positive added. If this ratio is unacceptable, then the selection rate should not be increased and consideration might be given to decreasing it. This doctor is behaving as if 33 were the ideal substitution rate. This would be optimal if the benefit of an additional true positive were 33 times greater than the cost of a false positive. We will not argue whether this benefit-cost ratio is correct or incorrect, because that is not a technical matter. The substitution rate is, however, a meaningful number, and informed social policy requires consideration of the substitution rate in the practice of mammography.

Mammography for women aged 50 or older is more accurate than for younger women (due to reduced breast density), and they have a higher base rate. This can result in a decrease in both false positives and false negatives, as shown in Table 2.

Table 3 compares the results for women under 50 and over 50, clearly showing why it is easier to recommend screening mammography for the over- 50 group. Even with fewer women selected for biopsy, both the false positive and false negative proportions are substantially lower for the older study sample.

Table 3 raises a question for this mammographer: Why should the marginal substitution rate be lower for women 50 and older than for women under 50 ? If the selection rate for women 50 and older were increased to 0.036 , the marginal substitution rate would be approximately equal to that for women under 50 (33.2) and the false negative proportion would drop to 0.135 , although the false positive proportion would increase to 0.029 . This is not to say that different marginal substitution rates for different age groups are necessarily inappropriate. For instance, more aggressive tumors or more life years at risk for younger women might justify more aggressive diagnostic practice for younger women. The point is that the substitution rate is almost certainly the result of intuitive processes. If these results were found to generalize to a larger sample of mammographers, health care consumers should decide, as a personal matter, and society should decide, as a policy matter, what tradeoffs are acceptable.

\footnotetext{
5 This calculation is based on signal detection theory and assumes that the distributions of the mammographer's judgments for both malignant and non-malignant cases are normal and both have the same standard deviation. For details of the calculation, contact the authors.
} 
Table 2. Results for women 50 years of age or older.

\begin{tabular}{llccr} 
& & \multicolumn{2}{c}{ Decision } & \\
& & Negative & Positive & \multicolumn{1}{c}{ Total } \\
\hline Biopsy & Malignant & 15 & 74 & 89 \\
result & Non-malignant & 9,704 & 207 & 9,911 \\
& Total & 9,719 & 281 & 10,000 \\
\hline
\end{tabular}

Based on Kolb, Lichy, and Newhouse (2002). Sample size adjusted to 10,000 for ease of interpretation.

Table 3. Comparison of women under 50 and those 50 years or older.

\begin{tabular}{lcccccc}
\hline Age & Base rate & $\mathrm{A}_{\mathrm{z}}$ & $\begin{array}{c}\text { Selection } \\
\text { Rate }\end{array}$ & $\begin{array}{c}\text { False } \\
\text { Positive } \\
\text { Proportion }\end{array}$ & $\begin{array}{c}\text { False } \\
\text { Negative } \\
\text { Proportion }\end{array}$ & $\begin{array}{c}\text { Imputed } \\
\text { Marginal } \\
\text { Substitution } \\
\text { Rate }\end{array}$ \\
\hline <50 years & 0.0086 & 0.90 & 0.0604 & 0.0559 & 0.4186 & 33.2 \\
$\geq 50$ years & 0.0089 & 0.98 & 0.0281 & 0.0209 & 0.1685 & 21.9 \\
\hline
\end{tabular}

\section{"WHAT-IF" QUESTIONS}

Table 4 examines two critical what-if questions regarding the practice of mammography:

1. What if the decision threshold/selection rate were changed? This is under the control of the radiologist, yet has important policy implications and implications for women and their families that are rarely discussed.

2. What if the accuracy of screening mammography were increased? Ways of increasing the accuracy of mammography are discussed below.

Rows 1 and 2 address the consequences of lowering the threshold (Question 1). In this case, the number of false positives doubles while the false negatives are reduced by one-third. The substitution rate doubles. Comparing rows 1 and 3 shows what happens when the threshold is increased. False positives decrease but false negatives increase, and the substitution rate decreases. Comparing row 2 with 4 and row 3 with 5 shows the effect of increasing the accuracy of mammography (Question 2). Both false positives and false negatives decrease. Rows 4 and 5 again illustrate the effect of raising the threshold. False positives decrease but false negatives increase. The substitution rate drops substantially.

As Table 4 illustrates, changing the threshold alone only trades one kind of error for another, and the marginal substitution rate quantifies that tradeoff. The analysis presents decisionmakers with a difficult dilemma: What is the appropriate substitution rate? Surveys might be one source of guidance. For example, a survey by Schwartz et al. (2000) found that 63 percent of the women sampled would accept 
Table 4. Comparison of mammographer performance under various what-if conditions for women under 50 .

\begin{tabular}{|c|c|c|c|c|c|c|}
\hline Age & Base rate & $A_{z}$ & $\begin{array}{c}\text { Selection } \\
\text { Rate }\end{array}$ & $\begin{array}{c}\text { False } \\
\text { Positive } \\
\text { (out of } \\
10,000 \text { ) }\end{array}$ & $\begin{array}{c}\text { False } \\
\text { Negative } \\
\text { (out of } \\
10,000 \text { ) }\end{array}$ & $\begin{array}{c}\text { Imputed } \\
\text { Marginal } \\
\text { Substitution } \\
\text { Rate }\end{array}$ \\
\hline 1. Kolb et al. data & 0.0086 & 0.90 & 0.0604 & 554 & 36 & 33.2 \\
\hline 2. Lower threshold & 0.0086 & 0.90 & 0.1200 & 1138 & 24 & 66.9 \\
\hline 3. Higher threshold & 0.0086 & 0.90 & 0.0500 & 453 & 39 & 27.9 \\
\hline \multirow{2}{*}{$\begin{array}{l}\text { and more accurate } \\
\text { 5. Higher threshold } \\
\text { and more accurate }\end{array}$} & 0.0086 & 0.95 & 0.1200 & 1125 & 11 & 103.9 \\
\hline & 0.0086 & 0.95 & 0.0500 & 437 & 23 & 32.6 \\
\hline
\end{tabular}

500 false positives to save one life. (This is not the same as our substitution rate because a true positive does not necessarily result in a life saved.)

Current practice might be another guide. Humphrey et al. (2002) calculated that "over 10 years of biennial screening among 40-year-old women invited to be screened, approximately 400 women would have false-positive results on mammography and 100 women would undergo biopsy or fine-needle aspiration for each death from breast cancer prevented" (p. 356). It is noteworthy that these results are consistent with the preferences expressed in the survey described above. Determination of the substitution rate should be a matter for policy debate.

Explicit recognition and discussion of the tradeoff between false positives and false negatives could give women greater control over the outcomes of mammography. Screening might be tailored to the values and risk preferences of specific patients or at least patient subgroups. If patients in group $X$ are more disturbed by biopsy than patients in group $\mathrm{Y}$, one might want to adopt more aggressive screening policies in group $\mathrm{Y}^{6}$

Increasing the accuracy of mammography reduces both kinds of error. There is no need to consider tradeoffs in choosing between rows 2 and 3 or between rows 1 and 4 above. In decision analytic terms, row 3 "dominates" row 2; that is, row 3 is better in all respects. Similarly, row 4 dominates row 1 .

A number of studies have investigated ways of improving the accuracy of mammographers' judgments. For example, Getty et al. (1988) found that by using a simple checklist that directed their attention to 12 important features in a mammogram, general radiologists could match the accuracy of experienced mammographers.

Accuracy may increase with the volume of mammograms read. Esserman et al. (2002) compared results in the United States with other countries, such as the United Kingdom and Sweden, that have high-volume, centralized screening programs. They found that for a fixed level of specificity, sensitivity was higher for U.K. radiologists than for U.S. radiologists, and the sensitivity of U.S. radiologists declined as the volume of mammograms read declined. They concluded that "reader volume is an important determinant of mammogram sensitivity and specificity." In an international study of mammography, Elmore et al. (2003) found that

6 Thanks to an anonymous reviewer for this insight. 
North American radiologists judged 2 to 4 percent more mammograms as abnormal than in other countries, without an increase in the number of cancers detected. A recent U.S.-U.K. comparison (Smith-Bindman et al., 2003) found similar results. Beam, Conant, and Sickles (2003), on the other hand, did not find this effect.

Several studies of computer aids for decision making in mammography have found promising results both for improving accuracy and reducing variability among radiologists (Chan et al., 1999; Floyd, Lo, and Tourassi, 2000; Haque, Mital, and Srinivasan, 2002; Jiang et al., 2001; Roque and Andre, 2002). There is also a strong likelihood that digital mammography will replace film in the future, and some evidence this will result in improved accuracy (Fischer et al., 2002; Nawano, 1995). Further, double reading of mammograms is a generally recognized method for improving accuracy (Beam, Conant, and Sickles, 2003; Karssemeijer et al., 2003; Kopans, 2000; Kwek et al., 2003; Liston and Dall, 2003). Kleit and Ruiz (2003) found that availability of previous mammogram reduced false positives by 50 percent.

Although improving accuracy reduces both kinds of error, there is still a tradeoff. The costs involved in methods for increasing accuracy must be justified by the benefits of reduced errors. Through a more complete analysis of the type illustrated here, it can be shown that methods for increasing the accuracy of mammography, even a little, will produce substantial benefits. Further analysis would be needed to determine whether the benefits justify the costs, but some of the methods described above, such as the use of a checklist, would seem to have low cost.

\section{FURTHER EXTENSIONS}

The method of analysis we have described is not new. Signal detection theory has been applied to many detection and selection problems, including mammography. Typically, such studies use the area under the ROC curve $\left(A_{z}\right)$ as a measure of accuracy that is independent of the decision threshold. What we have illustrated, and are proposing, differs from current practice in two ways.

First, performance data that include all four possible outcomes (Tables 1 and 2) can be used to estimate both accuracy and decision threshold. Such data are not routinely collected. Mammography regulations developed by the Food and Drug Administration under the Mammography Quality Standards Act (<http://www.fda.gov/cdrh/mammography/>) require audits based on only positive decisions (the decision to recommend biopsy). Therefore, data on false negatives and true negatives are not routinely available. There are good practical reasons for this. If a woman does not have a biopsy, follow-up is difficult. However, with improvements in medical record keeping, and the advent of electronic records, long-term follow-up becomes more feasible.

Second, while it is generally recognized that decision thresholds vary over time and among individuals, accuracy is often regarded as fixed. We have argued that accuracy can also be changed, and that interventions that address radiologists' judgment are likely to be relatively cheap and effective. It is important to conduct what-if analysis of both accuracy and decision threshold.

Finally, the approach that we have introduced here can be readily extended to address what-if questions related to mammography policy that do not focus narrowly on the practice of radiologists and mammographers. We can illustrate with two obvious extensions; many more examples could be generated:

1. What if the base rate for those who seek screening mammography were changed? The base rate might be changed in various ways, including recom- 
mending mammography only for older women, recommending mammography less frequently, and recommending mammography only for women with a family history of breast cancer.

2. What if we could reduce the costs of false positive results or increase the benefits of true positive diagnoses? If we could make biopsy less distasteful or costly, if we could explain the issues better to anxious women, or find other means to reduce the costs of false positive diagnoses, the low predictive value of screening mammography would exact lesser costs from women and their families. ${ }^{7}$ Improved treatment protocols that led to better prognoses with fewer noxious and debilitating side effects might likewise change the decision calculus.

The present example, involving screening mammography, illustrates clearly that the facts rarely if ever "speak for themselves" in policy problems that involve detection or selection decisions. Translating scientific or policy analytic studies so that they are made relevant to practice requires application of analytic techniques that are able to pose and answer counter-factual, what-if questions in meaningful ways.

We are grateful to Christine Muller for assistance with the literature review for this paper and to an anonymous reviewer for many helpful suggestions.

THOMAS R. STEWART is Research Professor at the Center for Policy Research, Rockefeller College of Public Affairs and Policy, University at Albany, State University of New York.

JERYL L. MUMPOWER is Interim Provost at the University at Albany, State University of New York. He is also Professor of Public Administration and Policy and Faculty Associate at the Center for Policy Research, Rockefeller College of Public Affairs and Policy, University at Albany.

\section{REFERENCES}

Beam, C.A., Conant, E.F., \& Sickles, E.A. (2003). Association of volume and volume-independent factors with accuracy in screening mammogram interpretation. Journal of the National Cancer Institute, 95(4), 282-290.

Beam, C.A., Layde, P.M., \& Sullivan, D.C. (1996). Variability in the interpretation of screening mammograms by US radiologists. Findings from a national sample. Archives of Internal Medicine, 156(2), 209-213.

Berg, W.A., Campassi, C., Langenberg, P., \& Sexton, M.J. (2000). Breast Imaging Reporting and Data System: Inter- and intraobserver variability in feature analysis and final assessment. American Journal of Roentgenology, 174(6), 1769-1777.

Berg, W.A., D’Orși, C.J., Jackson, V.P., Bassett, L.W., Beam, C.A., Lewis, R.S., \& Crewson, P.E. (2002). Does training in the Breast Imaging Reporting and Data System (BI-RADS) improve biopsy recommendations or feature analysis agreement with experienced breast imagers at mammography? Radiology, 224(3), 871-880.

Chan, H.P., Sahiner, B., Helvie, M.A., Petrick, N., Roubidoux, M.A., Wilson, T.E., Adler, D.D., Paramagul, C., Newman, J.S., \& Sanjay-Gopal, S. (1999). Improvement of radiologists'

${ }^{7}$ Thanks to an anonymous reviewer for this insight. 
characterization of mammographic masses by using computer-aided diagnosis: An ROC study. Radiology, 212(3), 817-827.

de Koning, H.J. (2003). Mammographic screening: Evidence from randomised controlled trials. Annals of Oncology, 14(8), 1185-1189.

Elmore, J.G., Barton, M.B., Moceri, V.M., Polk, S., Arena, P.J., \& Fletcher, S.W. (1998). Tenyear risk of false positive screening mammograms and clinical breast examinations. New England Journal of Medicine, 338(16), 1089-1096.

Elmore, J.G., Miglioretti, D.L., Reisch, L.M., Barton, M. B., Kreuter, W., Christiansen, C.L., \& Fletcher, S.W. (2002). Screening mammograms by community radiologists: Variability in false-positive rates. Journal of the National Cancer Institute, 94(18), 1373-1380.

Elmore, J.G., Nakano, C.Y., Koepsell, T.D., Desnick, L.M., D’Orsi, C.J., \& Ransohoff, D.F. (2003). International variation in screening mammography interpretations in community-based programs. Journal of the National Cancer Institute, 95(18), 1384-1393.

Esserman, L., Cowley, H., Eberle, C., Kirkpatrick, A., Chang, S., Berbaum, K., \& Gale, A. (2002). Improving the accuracy of mammography: Volume and outcome relationships. Journal of the National Cancer Institute, 94(5), 369-375.

Fischer, U., Baum, F., Obenauer, S., Luftner-Nagel, S., von Heyden, D., Vosshenrich, R., \& Grabbe, E. (2002). Comparative study in patients with microcalcifications: Full-field digital mammography vs screen-film mammography. European Radiology, 12(11), 2679-2683.

Floyd, C.E. Jr., Lo, J.Y., \& Tourassi, G.D. (2000). Case-based reasoning computer algorithm that uses mammographic findings for breast biopsy decisions. American Journal of Roentgenology, 175(5), 1347-1352.

Getty, D.J., Pickett, R.M., D’Orsi, C.J., \& Swets, J.A. (1988). Enhanced interpretation of diagnostic images. Investigations in Radiology, 23, 240-252.

Hammond, K.R., Harvey, L.O., \& Hastie, R. (1992). Making better use of scientific knowledge: Separating truth from justice. Psychological Science, 3(2), 80-87.

Haque, S., Mital, D., \& Srinivasan, S. (2002). Advances in biomedical informatics for the management of cancer. Annals of the New York Academy of Science, 980, 287-297.

Humphrey, L.L., Helfand, M., Chan, B.K., \& Woolf, S.H. (2002). Breast cancer screening: A summary of the evidence for the U.S. Preventive Services Task Force. Annals of Internal Medicine, 137(5 Part 1), 347-360.

Jiang, Y., Nishikawa, R.M., Schmidt, R.A., Toledano, A.Y., \& Doi, K. (2001). Potential of computer-aided diagnosis to reduce variability in radiologists' interpretations of mammograms depicting microcalcifications. Radiology, 220(3), 787-794.

Karssemeijer, N., Otten, J.D., Verbeek, A.L., Groenewoud, J.H., de Koning, H.J., Hendriks, J.H., \& Holland, R. (2003). Computer-aided detection versus independent double reading of masses on mammograms. Radiology, 227(1), 192-200.

Kleit, A.N., \& Ruiz, J.F. (2003). False positive mammograms and detection controlled estimation. Health Services Research, 38(4), 1207-1228.

Kolb, T.M., Lichy, J., \& Newhouse, J.H. (2002). Comparison of the performance of screening mammography, physical examination, and breast US and evaluation of factors that influence them: An analysis of 27,825 patient evaluations. Radiology, 225(1), 165-175.

Kopans, D.B. (2000). Double reading. Radiology Clinics of North America, 38(4), 719-724.

Kwek, B.H., Lau, T.N., Ng, F.C., \& Gao, F. (2003). Non-consensual double reading in the Singapore Breast Screening Project: Benefits and limitations. Annals of the Academy of Medicine, Singapore, 32(4), 438-441.

Liston, J.C., \& Dall, B.J. (2003). Can the NHS Breast Screening Programme afford not to double read screening mammograms? Clinical Radiology, 58(6), 474-477.

Mumpower, J.L., Nath, R., and Stewart, T.R. (2002). Affirmative action, duality of error, and 
the consequences of mispredicting the academic performance of African-American college applicants. Journal of Policy Analysis and Management, 21(1), 63-77.

Mushlin, A.I., Kouides, R.W., \& Shapiro, D.E. (1998). Estimating the accuracy of screening mammography: A meta-analysis. American Journal of Preventive Medicine, 14(2), 143-153.

National Breast Cancer Coalition (2003). Position statement on screening mammography, March 2003. Available at: <http://www.natlbcc.org/bin/index.asp?strid=560\&depid=9\& btnid=1 >; accessed June 7, 2004.

Nawano, S. (1995). Evaluation of digital mammography in diagnosis of breast cancer. Journal of Digital Imaging, 8(1 Suppl 1), 67-69.

Olsen, O., \& Gotzsche, P.C. (2001). Cochrane review on screening for breast cancer with mammography. Lancet, 358(9290), 1340-1342.

Pauker, S.G., \& Kassirer, J.P. (1980). The threshold approach to clinical decision making. New England Journal of Medicine, 302, 1109-1117.

Ries, L.A.G., Eisner, M.P., Kosary, C.L., Hankey, B.F., Miller, B.A., Clegg, L., Mariotto, A., Fay, M.P., Feuer, E.J., \& Edwards, B.K. (Eds.). (2003). SEER cancer statistics review, 1975-2000, National Cancer Institute. Bethesda, MD. Available at: <http://seer.cancer.gov/ csr/1975_2000>; accessed June 7, 2004.

Rimer, B.K., Halabi, S., Sugg Skinner, C., Lipkus, I.M., Strigo, T.S., Kaplan, E.B., \& Samsa, G.P. (2002). Effects of a mammography decision-making intervention at 12 and 24 months. American Journal of Preventive Medicine, 22(4), 247-257.

Roque, A.C., \& Andre, T.C. (2002). Mammography and computerized decision systems: A review. Annals of the New York Academy of Science, 980, 83-94.

Salzmann, P., Kerlikowske, K., \& Phillips, K. (1997). Cost-effectiveness of extending screening mammography guidelines to include women 40 to 49 years of age. Annals of Internal Medicine, 127(11), 955-965.

Schwartz, L.M., Woloshin, S., Black, W.C., \& Welch, H.G. (1997). The role of numeracy in understanding the benefit of screening mammography. Annals of Internal Medicine, 127, 966-972.

Schwartz, L.M., Woloshin, S., Sox, H.C., Fischhoff, B., \& Welch, H.G. (2000). US women's attitudes to false positive mammography results and detection of ductal carcinoma in situ: Cross sectional survey. British Medical Journal, 320(7250), 1635-1640.

Smith-Bindman, R., Chu, P.W., Miglioretti, D.L., Sickles, E.A., Blanks, R., Ballard-Barbash, R., Bobo, J.K., Lee, N.C., Wallis, M.G., Patnick, J., \& Kerlikowske, K. (2003). Comparison of screening mammography in the United States and the United Kingdom. Journal of the American Medical Association, 290(16), 2129-2137.

Swets, J.A., Dawes, R.M., \& Monahan, J. (2000). Psychological science can improve diagnostic decisions. Psychological Science in the Public Interest, 1(1), 1-26.

U.S. Preventive Services Task Force (2002). Guide to clinical preventive services, 3rd edition: Periodic updates: Screening for breast cancer. Available at: <http://www.ahcpr.gov/ clinic/uspstf/uspsbrca.htm>; accessed June 7, 2004.

Woolf, S.H. (2001). The accuracy and effectiveness of routine population screening with mammography, prostate-specific antigen, and prenatal ultrasound: A review of published scientific evidence. International Journal of Technology Assessment Health Care, 17(3), 275-304. 